\title{
Exact Solutions for a New Nonlinear KdV-Like Wave Equation Using Simplest Equation Method and Its Variants
}

\author{
Yinghui He, Yun-Mei Zhao, and Yao Long \\ Department of Mathematics, Honghe University, Mengzi, Yunnan 661100, China \\ Correspondence should be addressed to Yao Long; yaolong04@163.com
}

Received 7 February 2014; Accepted 9 June 2014; Published 25 June 2014

Academic Editor: Richard Saurel

Copyright (C) 2014 Yinghui He et al. This is an open access article distributed under the Creative Commons Attribution License, which permits unrestricted use, distribution, and reproduction in any medium, provided the original work is properly cited.

\begin{abstract}
The simplest equation method presents wide applicability to the handling of nonlinear wave equations. In this paper, we focus on the exact solution of a new nonlinear KdV-like wave equation by means of the simplest equation method, the modified simplest equation method and, the extended simplest equation method. The KdV-like wave equation was derived for solitary waves propagating on an interface (liquid-air) with wave motion induced by a harmonic forcing which is more appropriate for the study of thin film mass transfer. Thus finding the exact solutions of this equation is of great importance and interest. By these three methods, many new exact solutions of this equation are obtained.
\end{abstract}

\section{Introduction}

Nonlinear phenomena play a crucial role in applied mathematics and physics. Calculating exact and numerical solutions, in particular the traveling wave solutions of nonlinear equations in mathematical physics, plays an important role in soliton theory. In the recent years, much effort has been spent on this task and many significant methods have been established such as inverse scattering transform [1], Bäcklund and Darboux transform [2], Hirota method [3], homogeneous balance method [4], Jacobi elliptic function method [5], tanh-function method [6], Exp-function method [7], $G^{\prime} / G$ expansion method $[8,9]$, the method for construction of meromorphic solutions [10,11], variational iteration method [12-14], and He's homotopy perturbation method [15-19].

Partially, the simplest equation method is a very powerful mathematical technique to seek more new solutions of NLEEs that can be expressed as polynomial in an elementary function which satisfies a subequation like Riccati equation, auxiliary ordinary equation, elliptic equation, and generalized Riccati equation. It has been developed by Fan [20] and Kudryashov [21] and the method optimizes the use of the auxiliary equation and effectively avoids producing some duplication solutions. There are many applications and generalizations of the method [22-25].
Recently, Bilige introduced a method called the extended simplest equation method as an extension of the simplest equation method, to look for the exact traveling wave solutions of nonlinear evolution equations (NLEEs) [26, 27]. In this method, a second order linear ordinary differential equation (ODE) is taken as the auxiliary equation. This method can construct different forms of exact traveling wave solutions which cannot be obtained by using the tanh-function method, F-expansion method, and the Expfunction method.

The nonlinear shallow water surface waves satisfy the Korteweg-de Vries (KdV) equation:

$$
w_{t}+c_{0} w_{x}+a_{1} w w_{x}+a_{2} w_{x x x}=0 .
$$

This equation is only valid for long waves. Solitary waves in film flows were studied by $[18,19]$. These flows also show a transition to turbulence. This process is better understood, if the dynamics of nonlinear waves are traceable.

In [28] Rees and Zimmerman derived a new nonlinear KdV-like evolution equation for surface solitary waves propagating on a liquid-air interface where the wave motion is induced by harmonic forcing:

$$
w_{x x x}+k^{2} w_{x}+\epsilon c \lambda_{3}\left(w_{x}^{2}-w w_{x x}\right)-\frac{2 k^{2}}{c} w_{t}=0 .
$$


The nonlinearity term $w w_{x}$ in (1) does not appear in (2), and the reconstruction of this term from $\left(w_{x}^{2}-w w_{x x}\right)$ is not possible by order approximation equivalences; also the nonlinearity term in (2) is more steep.

It was observed by Sohail et al. [29] that wave data obtained from an online measurement technique satisfies this evolution equation, when the wave length is just a few multiples of the fluid depth. In [30], the integrability of this new nonlinear partial differential equation was discussed with a focus on the Painleve property, the compatibility condition, and the Bäcklund transformation.

The exact traveling wave solutions of (2) are not reported in related references. We have tried many other algebraic methods to seek exact solutions of (2). However, we find that they are either not efficient or invalid. In this work, we apply the simplest equation method, the modified simplest equation method, and the extended simplest equation method for obtaining exact traveling solutions of (2). From the results we see that these methods are more effective.

The organization of the paper is as follows. In Section 2, a brief description of three simplest equation methods for finding traveling wave solutions of nonlinear equations is given. In Section 3, we will study (2) by these methods. Finally conclusions are given in Section 4.

\section{Description of the Simplest Equation Methods}

\subsection{The Simplest Equation Method}

Step 1. Consider a general nonlinear PDE in the form

$$
F\left(u, u_{x}, u_{t}, u_{x x}, u_{x t}, \ldots\right)=0
$$

Using $u(x, t)=\phi(\xi), \xi=\alpha x+\beta t$, we can rewrite (6) as the following nonlinear ODE:

$$
F\left(\phi, \phi^{\prime}, \phi^{\prime \prime}, \ldots\right)=0,
$$

where the prime denotes differentiation with respect to $\xi$.

Step 2. Suppose that the solution of ODE (4) can be written as follows:

$$
\phi(\xi)=\sum_{i=1}^{n} A_{i} f^{i}(\xi)
$$

where $f(\xi)$ are the functions that satisfy some ordinary differential equations. The simplest equation has two properties: first it is of lesser order than (4) and second we know the general solution of the simplest equation. $n$ is a positive integer that can be determined by balancing procedure, and $A_{i}(i=0,1,2, \ldots)$ are parameters to be determined.

In this paper, we will use as simplest equation the equations of Bernoulli and Riccati which are well known nonlinear ordinary differential equations and their solutions can be expressed by elementary functions.

For the Bernoulli equation

$$
f^{\prime}(\xi)=a f(\xi)+b f^{2}(\xi),
$$

equation (6) admits the following exact solutions:

$$
f(\xi)=\frac{a \exp \left(a\left(\xi+\xi_{0}\right)\right)}{1-b \exp \left(a\left(\xi+\xi_{0}\right)\right)}
$$

for the case $a>0, b<0$ and

$$
f(\xi)=-\frac{a \exp \left(a\left(\xi+\xi_{0}\right)\right)}{1+b \exp \left(a\left(\xi+\xi_{0}\right)\right)}
$$

for the case $a<0, b>0$, where $\xi_{0}$ is a constant.

For the Riccati equation

$$
f^{\prime}(\xi)=a f^{2}(\xi)+b .
$$

Equation (9) admits the following exact solutions:

$$
f(\xi)=-\frac{\sqrt{-a b}}{a} \tanh \left(\sqrt{-a b} \xi-\frac{\varepsilon \ln \xi_{0}}{2}\right), \quad \varepsilon= \pm 1,
$$

when $a b<0$ and

$$
f(\xi)=\frac{\sqrt{a b}}{a} \tan \left(\sqrt{a b} \xi+\xi_{0}\right)
$$

when $a b>0$, where $\xi_{0}$ is a constant.

Step 3. Substituting (5) along with (6) (or (9)) into (4), then the left-hand side of (4) is converted into a polynomial in $f(\xi)$; equating each coefficient of the polynomial to zero yields a set of algebraic equations for $A_{i}, a, b$.

Step 4. Solving the algebraic equations obtained in Step 3 and substituting the results into (5), we obtain the exact traveling wave solutions for (3).

2.2. The Modified Simplest Equation Method. In the modified version, one makes an ansatz for the solution $\phi(\xi)$ as

$$
\phi(\xi)=\sum_{i=0}^{n} A_{i}\left(\frac{\varphi^{\prime}}{\varphi}\right)^{i}
$$

where $A_{i}(i=0,1,2, \ldots, n)$ are arbitrary constants to be determined, such that $a_{n} \neq 0$ and $\varphi=\varphi(\xi)$ is an unspecified function to be determined afterward.

Substitute (12) into (4) and then we account the function $\varphi$. As a result of this substitution, we get a polynomial of $\varphi^{\prime} / \varphi$ and its derivatives. In this polynomial, we equate the coefficients of the same power of $\varphi^{-j}$ to zero, where $j>0$. This procedure yields a system of equations which can be solved to find $A_{i}(i=0,1,2, \ldots, n), \varphi$, and $\varphi^{\prime}$. Then the substitution of the results into (12) completes the determination of exact solutions of (3).

2.3. The Extended Simplest Equation Method. We suppose that the solutions for (4) can be expressed in the following form:

$$
\phi(\xi)=\sum_{j_{1}=0}^{n} A_{j_{1}}\left(\frac{\varphi^{\prime}}{\varphi}\right)^{j_{1}}+\sum_{j_{2}=0}^{n-1} B_{j_{2}}\left(\frac{\varphi^{\prime}}{\varphi}\right)^{j_{2}} \frac{1}{\varphi},
$$


where $A_{j_{1}}, B_{j_{2}}\left(j_{1}=0,1, \ldots, n ; j_{2}=0,1, \ldots, n-1\right)$ are constants and $A_{n} B_{n-1} \neq 0$. The function $\varphi=\varphi(\xi)$ satisfies the second order linear ODE in the form

$$
\varphi^{\prime \prime}+\delta \varphi=\nu
$$

where $\delta$ and $v$ are constants. Equation (14) has three types of general solution with double arbitrary parameters as follows:

$$
\begin{aligned}
& \varphi(\xi) \\
& = \begin{cases}C_{1} \cosh (\sqrt{-\delta} \xi)+C_{2} \sinh (\sqrt{-\delta} \xi)+\frac{\nu}{\delta}, & \delta<0, \\
C_{1} \cos (\sqrt{\delta} \xi)+C_{2} \sin (\sqrt{\delta} \xi)+\frac{\nu}{\delta}, & \delta>0, \\
\frac{\nu}{2} \xi^{2}+C_{1} \xi+C_{2}, & \delta=0,\end{cases} \\
& \left(\frac{\varphi^{\prime}}{\varphi}\right)^{2}= \begin{cases}\left(\delta C_{1}^{2}-\delta C_{2}^{2}-\frac{\nu^{2}}{\delta}\right)\left(\frac{1}{\varphi}\right)^{2}-\delta+\frac{2 \nu}{\varphi}, & \delta<0, \\
\left(\delta C_{1}^{2}+\delta C_{2}^{2}-\frac{\nu^{2}}{\delta}\right)\left(\frac{1}{\varphi}\right)^{2}-\delta+\frac{2 \nu}{\varphi}, & \delta>0, \\
\left(C_{1}^{2}-2 \nu C_{2}\right)\left(\frac{1}{\varphi}\right)^{2}+\frac{2 \nu}{\varphi}, & \delta=0,\end{cases}
\end{aligned}
$$

where $C_{1}, C_{2}$ are arbitrary constants.

By substituting (13) into (4) and using the second order linear ODE (14) and (16), collecting all terms with the same order of $1 / \varphi^{i}$ and $\left(1 / \varphi^{i}\right)\left(\varphi^{\prime} / \varphi\right)$ together, the left-hand side of (4) is converted into another polynomial in $1 / \varphi^{i}$ and $\left(1 / \varphi^{i}\right)\left(\varphi^{\prime} / \varphi\right)$. Equating each coefficient of these different power terms to zero yields a set of algebraic equations for $A_{i}, B_{j}(i=0,1, \ldots, n, j=0,1, \ldots, n-1), \delta$, and $v$.

Assuming constants $A_{i}, B_{j}(i=0,1, \ldots, n, j=0,1, \ldots$, $n-1), \delta$, and $\nu$ can be determined by solving the algebraic equations, then substituting these terms and the general solutions (15) of (14) into (13), we have many exact traveling wave solutions of the NLEE (3).

\section{Exact Solutions of New KdV-Like Equation}

Making a transformation $w(x, t)=\phi(\xi)$ with $\xi=\alpha x+\beta t$, (2) can be reduced to the following ODE:

$$
\alpha^{3} \phi^{\prime \prime \prime}+k^{2} \alpha \phi^{\prime}+\epsilon c \lambda_{3}\left(\alpha^{2} \phi^{\prime 2}-\alpha^{2} \phi \phi^{\prime \prime}\right)-\frac{2 k^{2} \beta}{c} \phi^{\prime}=0,
$$

where $\alpha, \beta, k, c, \epsilon$, and $\lambda_{3}$ are nonzero constants. Balancing $\phi^{\prime \prime \prime}$ and $\phi \phi^{\prime \prime}$ in (17), we obtain $n+3=n+n+2$ which gives $n=1$.

3.1. Using Simplest Equation Method. Suppose that (17) owns the solutions in the form

$$
\phi(\xi)=A_{0}+A_{1} f(\xi) .
$$

For the Bernoulli equation, substituting (18) into (17) and making use of the Bernoulli equation (6) and then setting all the coefficients of $f^{k}(\xi)$ of the resulting system to zero, we obtain an algebraic system of equations in terms of $A_{i}(i=0,1), a, b, \alpha$, and $\beta$. Solving this system of algebraic equations, we obtain

$$
\begin{gathered}
A_{0}=\frac{3 \alpha a}{\epsilon c \lambda_{3}}, \quad A_{1}=\frac{6 \alpha b}{\epsilon c \lambda_{3}}, \quad \alpha=\alpha, \\
\beta=-\frac{c \alpha\left(2 \alpha^{2} a^{2}-k^{2}\right)}{2 k^{2}} .
\end{gathered}
$$

Therefore, using solutions (7) and (8) of (6) and ansatz (18), we obtain the following exact solution of (2):

$$
\begin{aligned}
w_{1}(x, t) & =\frac{3 \alpha a}{\epsilon c \lambda_{3}}+\frac{6 \alpha b}{\epsilon c \lambda_{3}} \frac{a \exp \left(a\left(\xi+\xi_{0}\right)\right)}{1-b \exp \left(a\left(\xi+\xi_{0}\right)\right)} \\
& =\frac{3 \alpha a}{\epsilon c \lambda_{3}}\left(1+\frac{2 b \exp \left(a\left(\xi+\xi_{0}\right)\right)}{1-b \exp \left(a\left(\xi+\xi_{0}\right)\right)}\right)
\end{aligned}
$$

for the case $a>0, b<0$ and

$$
\begin{aligned}
w_{2}(x, t) & =\frac{3 \alpha a}{\epsilon c \lambda_{3}}-\frac{6 \alpha b}{\epsilon c \lambda_{3}} \frac{a \exp \left(a\left(\xi+\xi_{0}\right)\right)}{1+b \exp \left(a\left(\xi+\xi_{0}\right)\right)} \\
& =\frac{3 \alpha a}{\epsilon c \lambda_{3}}\left(1-\frac{2 b \exp \left(a\left(\xi+\xi_{0}\right)\right)}{1+b \exp \left(a\left(\xi+\xi_{0}\right)\right)}\right)
\end{aligned}
$$

for the case $a<0, b>0$, where $\xi=\alpha x-\left(c \alpha\left(2 \alpha^{2} a^{2}-k^{2}\right) /\right.$ $\left.2 k^{2}\right) t$.

For the Riccati equation, substituting (18) into (17) and making use of the Riccati equation (9) and then setting all the coefficients of $f^{k}(\xi)$ of the resulting system to zero, we obtain an algebraic system of equations in terms of $A_{i}(i=0,1), a, b, \alpha$, and $\beta$. Solving this system of algebraic equations, we obtain

$$
\begin{gathered}
A_{0}=0, \quad A_{1}=\frac{6 \alpha a}{\epsilon c \lambda_{3}}, \quad \alpha=\alpha, \\
\beta=\frac{\alpha c\left(8 \alpha^{2} a b+k^{2}\right)}{2 k^{2}} .
\end{gathered}
$$

Therefore, using solutions (10) and (11) of (9) and ansatz (18), we obtain the following exact solution of (2):

$$
w_{3}(x, t)=-\frac{6 \alpha \sqrt{-a b}}{\epsilon c \lambda_{3}} \tanh \left(\sqrt{-a b} \xi-\frac{\varepsilon \ln \xi_{0}}{2}\right),
$$

for the case $a b<0$, and

$$
w_{4}(x, t)=\frac{6 \alpha \sqrt{a b}}{\epsilon c \lambda_{3}} \tan \left(\sqrt{a b} \xi+\xi_{0}\right),
$$

for the case $a b>0$, where $\xi=\alpha x+\left(\alpha c\left(8 \alpha^{2} a b+k^{2}\right) / 2 k^{2}\right) t$.

3.2. Using the Modified Simplest Equation Method. Suppose that (17) owns the solutions in the form

$$
\phi(\xi)=A_{0}+A_{1} \frac{\varphi^{\prime}}{\varphi}
$$


where $A_{0}$ and $A_{1}$ are constants, such that $A_{1} \neq 0$, and $\varphi=$ $\varphi(\xi)$ is an unspecified function to be determined. It is simple to calculate that

$$
\begin{aligned}
\phi^{\prime}= & A_{1}\left(\frac{\phi^{\prime \prime}}{\varphi}-\left(\frac{\varphi^{\prime}}{\varphi}\right)^{2}\right), \\
\phi^{\prime \prime}= & A_{1} \frac{\varphi^{\prime \prime \prime}}{\varphi}-3 A_{1} \frac{\varphi^{\prime \prime} \varphi^{\prime}}{\varphi^{2}}+2 A_{1}\left(\frac{\varphi^{\prime}}{\varphi}\right)^{3}, \\
\phi^{\prime \prime \prime}= & A_{1} \frac{\varphi^{(4)}}{\varphi}-A_{1} \frac{4 \varphi^{\prime \prime \prime} \varphi^{\prime}+3 \varphi^{\prime \prime 2}}{\varphi^{2}}+12 A_{1} \frac{\varphi^{\prime \prime} \varphi^{\prime 2}}{\varphi^{3}} \\
& -6 A_{1}\left(\frac{\varphi^{\prime}}{\varphi}\right)^{4},
\end{aligned}
$$

substituting (25) and (26) into (17) and equating the coefficients of $\varphi^{j}(j=-3,-2,-1,0)$ to zero yield the following system:

$$
\begin{aligned}
\varphi^{0}: & -A_{1} \alpha^{2} c \varphi^{\prime 4}\left(6 \alpha+\epsilon c \lambda_{3} A_{1}\right)=0, \\
\varphi^{-1}: A_{1}( & \left.k^{2} \alpha c \varphi^{\prime \prime}-2 k^{2} \beta \varphi^{\prime \prime}-\epsilon c^{2} \lambda_{3} \alpha^{2} A_{0} \varphi^{\prime \prime \prime}+\alpha^{3} c \varphi^{(4)}\right)=0, \\
\varphi^{-2}: A_{1}( & -3 \alpha^{3} c \varphi^{\prime \prime 2}-4 \alpha^{3} c \varphi^{\prime \prime \prime} \varphi^{\prime}-k^{2} \alpha c \varphi^{\prime 2}+\epsilon c^{2} \lambda_{3} \alpha^{2} A_{1} \varphi^{\prime \prime 2} \\
& +3 \epsilon c^{2} \lambda_{3} \alpha^{2} A_{0} \varphi^{\prime \prime} \varphi^{\prime}-\epsilon c^{2} \lambda_{3} \alpha^{2} A_{1} \varphi^{\prime \prime \prime} \varphi^{\prime} \\
& \left.+2 k^{2} \beta \varphi^{\prime 2}\right)=0,
\end{aligned}
$$$$
\varphi^{-3}: A_{1} \alpha^{2} c \varphi^{\prime 2}\left(12 \alpha \varphi^{\prime \prime}+\epsilon c \lambda_{3} A_{1} \varphi^{\prime \prime}-2 \epsilon c \lambda_{3} A_{0} \varphi^{\prime}\right)=0 .
$$

Solving the first equation of system (27), one has $A_{1}=0$ and $A_{1}=-6 \alpha / \epsilon c \lambda_{3}$. When $A_{1}=0$, we obtain trivial solution; therefore, the case is rejected. Substituting $A_{1}=-6 \alpha / \epsilon c \lambda_{3}$ into the other equations of system (27), we can obtain the following results:

$$
A_{0}=0, \quad \alpha=\frac{2 \beta}{c}, \quad \beta=\beta, \quad \varphi=k_{1} \xi+k_{2},
$$

where $k_{1}$ and $k_{2}$ are arbitrary constants, and

$$
\begin{gathered}
A_{0}= \pm \frac{3 k \sqrt{2 \alpha c(\alpha c-2 \beta)}}{2 \alpha c^{2} \epsilon \lambda_{3}}, \quad \alpha=\alpha, \\
\beta=\beta, \quad \varphi=k_{1}+k_{2} \exp \left(\frac{k \sqrt{2 \alpha c(\alpha c-2 \beta)}}{2 \alpha^{2} c} \xi\right),
\end{gathered}
$$

where $k_{1}$ and $k_{2}$ are arbitrary constants, $2 \alpha c(\alpha c-2 \beta)>0$.

Substituting (28) into (25), we can get the exact solution of (2):

$$
w_{5}(x, t)=A_{1} \frac{\varphi^{\prime}}{\varphi}=-\frac{12 \beta k_{1}}{c^{2} \epsilon \lambda_{3}\left(k_{1} \xi+k_{2}\right)},
$$

where $\xi=(2 \beta / c) x+\beta t$.
Substituting (29) into (25), we can get the exact solution of (2):

$$
\begin{aligned}
w_{6}(x, t) & A_{0}+A_{1} \frac{\varphi^{\prime}}{\varphi} \\
= & \frac{3 k \varepsilon \sqrt{2 \alpha c(\alpha c-2 \beta)}}{2 \epsilon c^{2} \lambda_{3}} \\
& \times\left(\frac{-k_{1}+k_{2} \exp \left(\left(-k \varepsilon \sqrt{2 \alpha c(\alpha c-2 \beta)} / 2 \alpha^{2} c\right) \xi\right)}{k_{1}+k_{2} \exp \left(\left(-k \varepsilon \sqrt{2 \alpha c(\alpha c-2 \beta)} / 2 \alpha^{2} c\right) \xi\right)}\right),
\end{aligned}
$$

where $\xi=\alpha x+\beta t, \varepsilon= \pm 1$.

We can arbitrarily choose the parameters $k_{1}$ and $k_{2}$. Therefore, if we set $k_{1}= \pm k_{2}$, (31) reduces to

$$
\begin{aligned}
w_{7}(x, t)= & -\frac{3 k \sqrt{2 \alpha c(\alpha c-2 \beta)}}{2 \epsilon c^{2} \lambda_{3}} \\
& \times \tanh \left(\frac{k \sqrt{2 \alpha c(\alpha c-2 \beta)}}{4 \alpha^{2} c} \xi\right), \\
w_{7}(x, t)= & \left.-\frac{3 k \sqrt{2 \alpha c(\alpha c-2 \beta)}}{2 \epsilon c^{2} \lambda_{3}}\right) \\
& \times \operatorname{coth}\left(\frac{k \sqrt{2 \alpha c(\alpha c-2 \beta)}}{4 \alpha^{2} c} \xi\right),
\end{aligned}
$$

where $2 \alpha c(\alpha c-2 \beta)>0$.

Using hyperbolic function identities, from (32), we obtain the following periodic solutions:

$$
\begin{aligned}
w_{9}(x, t)= & \frac{3 k \sqrt{-2 \alpha c(\alpha c-2 \beta)}}{2 \epsilon c^{2} \lambda_{3}} \\
& \times \tan \left(\frac{k \sqrt{-2 \alpha c(\alpha c-2 \beta)}}{4 \alpha^{2} c} \xi\right), \\
w_{10}(x, t)= & -\frac{3 k \sqrt{-2 \alpha c(\alpha c-2 \beta)}}{2 \epsilon c^{2} \lambda_{3}} \\
& \times \cot \left(\frac{k \sqrt{-2 \alpha c(\alpha c-2 \beta)}}{4 \alpha^{2} c}\right) .
\end{aligned}
$$


3.3. Using the Extended Simplest Equation Method. Suppose that (17) owns the solutions in the form

$$
\phi(\xi)=A_{0}+A_{1} \frac{\varphi^{\prime}}{\varphi}+B_{1} \frac{1}{\varphi},
$$

where $A_{0}, A_{1}$, and $B_{1}$ are constants to be determined later, $A_{1} A_{3} \neq 0$, and function $\varphi=\varphi(\xi)$ satisfies the second order linear ODE (14).

By substituting (34) into (17), using the second order linear ODE expressions (14) and (16), collecting all terms with the same order of $1 / \varphi^{i}$ and $\left(1 / \varphi^{i}\right)\left(\varphi^{\prime} / \varphi\right)$ together, the lefthand side of (17) is converted into another polynomial in $1 / \varphi^{i}$ and $\left(1 / \varphi^{i}\right)\left(\varphi^{\prime} / \varphi\right)$. Equating each coefficient of these different power terms to zero yields a set of algebraic equations for $A_{0}, A_{1}, B_{1}, \alpha, \beta, \delta$, and $\nu$. Solving these equations, we obtain the following results.

If $\delta>0$, one has

$$
\begin{gathered}
A_{0}=0, \quad A_{1}= \pm \sqrt{\frac{\delta}{\Delta}} B_{1}, \quad B_{1}=B_{1}, \\
\alpha= \pm \frac{1}{3} c \epsilon \lambda_{3} \sqrt{\frac{\delta}{\Delta}} B_{1}, \quad \delta=\delta, \quad \nu=\nu, \\
\beta= \pm\left(\left(\left(9 \delta^{2} k^{2} C_{2}^{2}-9 k^{2} \nu^{2}+9 \delta^{2} k^{2} C_{1}^{2}\right.\right.\right. \\
\left.\left.\left.+2 B_{1}^{2} \delta^{2} c^{2} \epsilon^{2} \lambda_{3}^{2}\right) c^{2} \in \lambda_{3} \sqrt{\frac{\delta}{\Delta}}\right)\left(54 \Delta k^{2}\right)^{-1}\right) B_{1},
\end{gathered}
$$

where $\Delta=\delta^{2} C_{1}^{2}+\delta^{2} C_{2}^{2}-\nu^{2}>0$, and

$$
\begin{aligned}
& A_{0}=0, \quad A_{1}=A_{1}, \quad B_{1}=0, \\
& \alpha=-\frac{1}{3} c \epsilon \lambda_{3} A_{1}, \quad \delta= \pm \frac{v}{\sqrt{C_{1}^{2}+C_{2}^{2}}}, \quad \nu=\nu, \\
& \beta=-\frac{c^{2} \epsilon \lambda_{3}}{54 k^{2}}\left( \pm \frac{2 c^{2} \epsilon^{2} \lambda_{3}^{2} A_{1}^{2} \nu}{\sqrt{C_{1}^{2}+C_{2}^{2}}}+9 k^{2}\right) A_{1}, \\
& A_{0}=0, \quad A_{1}=A_{1}, \quad B_{1}=0, \quad \alpha=-\frac{1}{6} c \in \lambda_{3} A_{1}, \\
& \beta=-\frac{c^{2} \epsilon \lambda_{3}\left(2 c^{2} \epsilon^{2} \lambda_{3}^{2} A_{1}^{2} \delta+9 k^{2}\right)}{108 k^{2}} A_{1}, \\
& \delta=\delta, \quad v=0, \\
& A_{0}=0, \quad A_{1}=A_{1}, \quad B_{1}=B_{1}, \\
& \alpha=-\frac{1}{3} c \epsilon \lambda_{3} A_{1}, \quad \delta=\frac{B_{1}^{2}}{A_{1}^{2}\left(C_{1}^{2}+C_{2}^{2}\right)}, \quad \nu=0,
\end{aligned}
$$

$$
\beta=-\frac{c^{2} \epsilon \lambda_{3}\left(2 c^{2} \epsilon^{2} \lambda_{3}^{2} B_{1}^{2}+9 k^{2} C_{1}^{2}+9 k^{2} C_{2}^{2}\right)}{54 k^{2}\left(C_{1}^{2}+C_{2}^{2}\right)} A_{1} .
$$

Substituting (35)-(38) into (34), making use of solutions (15) of (14), we can obtain, respectively, exact traveling wave solutions expressed by trigonometric functions of (2).

Consider

$$
\begin{aligned}
w_{11}(x, t)= & \pm \frac{\delta B_{1}\left(-C_{1} \sin (\sqrt{\delta} \xi)+C_{2} \cos (\sqrt{\delta} \xi)\right)}{\sqrt{\Delta}\left(C_{1} \cos (\sqrt{\delta} \xi)+C_{2} \sin (\sqrt{\delta} \xi)+(\nu / \delta)\right)} \\
& +\frac{B_{1}}{C_{1} \cos (\sqrt{\delta} \xi)+C_{2} \sin (\sqrt{\delta} \xi)+(\nu / \delta)},
\end{aligned}
$$

where $\xi=\alpha x+\beta t ; \alpha, \beta$ are determined in (35); $B_{1}, C_{1}, C_{2}, \delta$, and $\nu$ are arbitrary constants.

Consider

$$
w_{12}(x, t)=\frac{A_{1} \sqrt{\delta}\left(-C_{1} \sin (\sqrt{\delta} \xi)+C_{2} \cos (\sqrt{\delta} \xi)\right)}{C_{1} \cos (\sqrt{\delta} \xi)+C_{2} \sin (\sqrt{\delta} \xi)+(\nu / \delta)},
$$

where $\xi=\alpha x+\beta t ; \alpha, \beta, \delta$ are determined in (36); $A_{1}, C_{1}$, and $C_{2}$, and $v$ are arbitrary constants.

Consider

$$
w_{13}(x, t)=\frac{A_{1} \sqrt{\delta}\left(-C_{1} \sin (\sqrt{\delta} \xi)+C_{2} \cos (\sqrt{\delta} \xi)\right)}{C_{1} \cos (\sqrt{\delta} \xi)+C_{2} \sin (\sqrt{\delta} \xi)},
$$

where $\xi=\alpha x+\beta t ; \alpha, \beta$ are determined in (37); $A_{1}, C_{1}, C_{2}$, and $\delta$ are arbitrary constants.

Consider

$$
\begin{aligned}
w_{14}(x, t)= & \frac{A_{1} \sqrt{\delta}\left(-C_{1} \sin (\sqrt{\delta} \xi)+C_{2} \cos (\sqrt{\delta} \xi)\right)}{C_{1} \cos (\sqrt{\delta} \xi)+C_{2} \sin (\sqrt{\delta} \xi)} \\
& +\frac{B_{1}}{C_{1} \cos (\sqrt{\delta} \xi)+C_{2} \sin (\sqrt{\delta} \xi)},
\end{aligned}
$$

where $\xi=\alpha x+\beta t ; \alpha, \beta, \delta$ are determined in (38); $A_{1}, B_{1}$, and $C_{1}$, and $C_{2}$ are arbitrary constants.

If $\delta<0$, one has

$$
\begin{gathered}
A_{0}=0, \quad A_{1}= \pm \sqrt{\frac{-\delta}{\Delta}} B_{1}, \quad B_{1}=B_{1}, \\
\alpha= \pm \frac{1}{3} c \epsilon \lambda_{3} \sqrt{\frac{-\delta}{\Delta}} B_{1}, \quad \delta=\delta, \quad \nu=v, \\
\beta= \pm\left(\left(\left(-9 \delta^{2} k^{2} C_{2}^{2}-9 k^{2} \nu^{2}+9 \delta^{2} k^{2} C_{1}^{2}\right.\right.\right. \\
\left.\left.\left.+2 B_{1}^{2} \delta^{2} c^{2} \epsilon^{2} \lambda_{3}^{2}\right) c^{2} \epsilon \lambda_{3} \sqrt{\frac{-\delta}{\Delta}}\right)\left(54 \Delta k^{2}\right)^{-1}\right) B_{1},
\end{gathered}
$$

where $\Delta=-\delta^{2} C_{1}^{2}+\delta^{2} C_{2}^{2}+\nu^{2}>0$. 
Consider

$$
\begin{aligned}
& A_{0}=0, \quad A_{1}=A_{1}, \quad B_{1}=0, \\
& \alpha=-\frac{1}{3} c \epsilon \lambda_{3} A_{1}, \quad \delta= \pm \frac{v}{\sqrt{C_{2}^{2}-C_{1}^{2}}}, \quad \nu=\nu, \\
& \beta=-\frac{c^{2} \epsilon \lambda_{3}}{54 k^{2}}\left( \pm \frac{2 c^{2} \epsilon^{2} \lambda_{3}^{2} A_{1}^{2} \nu}{\sqrt{C_{2}^{2}-C_{1}^{2}}}+9 k^{2}\right) A_{1} \text {, } \\
& A_{0}=0, \quad A_{1}=A_{1}, \quad B_{1}=0, \quad \alpha=-\frac{1}{6} c \epsilon \lambda_{3} A_{1}, \\
& \beta=-\frac{c^{2} \epsilon \lambda_{3}\left(2 c^{2} \epsilon^{2} \lambda_{3}^{2} A_{1}^{2} \delta+9 k^{2}\right)}{108 k^{2}} A_{1} \text {, } \\
& \delta=\delta, \quad \nu=0, \\
& A_{0}=0, \quad A_{1}=A_{1}, \quad B_{1}=B_{1}, \\
& \alpha=-\frac{1}{3} c \epsilon \lambda_{3} A_{1}, \quad \delta=-\frac{B_{1}^{2}}{A_{1}^{2}\left(C_{2}^{2}-C_{1}^{2}\right)}, \quad \nu=0, \\
& \beta=\frac{c^{2} \epsilon \lambda_{3}\left(2 c^{2} \epsilon^{2} \lambda_{3}^{2} B_{1}^{2}+9 k^{2} C_{1}^{2}-9 k^{2} C_{2}^{2}\right)}{54 k^{2}\left(C_{2}^{2}-C_{1}^{2}\right)} A_{1} .
\end{aligned}
$$

Substituting (43)-(46) into (34), making use of solutions (15) of (14), we can obtain, respectively, exact traveling wave solutions expressed by hyperbolic functions of (2).

Consider

$$
\begin{aligned}
w_{15}(x, t) & \\
= & \pm \frac{\delta B_{1}\left(C_{1} \sinh (\sqrt{-\delta} \xi)+C_{2} \cosh (\sqrt{-\delta} \xi)\right)}{\sqrt{\Delta}\left(C_{1} \cosh (\sqrt{-\delta} \xi)+C_{2} \sinh (\sqrt{-\delta} \xi)+(\nu / \delta)\right)} \\
& +\frac{B_{1}}{C_{1} \cosh (\sqrt{-\delta} \xi)+C_{2} \sinh (\sqrt{-\delta} \xi)+(\nu / \delta)}
\end{aligned}
$$

where $\xi=\alpha x+\beta t ; \alpha, \beta$ are determined in (43); $B_{1}, C_{1}, C_{2}, \delta$, and $\nu$ are arbitrary constants.

Consider

$$
\begin{aligned}
& w_{16}(x, t) \\
& =\frac{A_{1} \sqrt{-\delta}\left(C_{1} \sinh (\sqrt{-\delta} \xi)+C_{2} \cosh (\sqrt{-\delta} \xi)\right)}{C_{1} \cosh (\sqrt{-\delta} \xi)+C_{2} \sinh (\sqrt{-\delta} \xi)+(\nu / \delta)},
\end{aligned}
$$

where $\xi=\alpha x+\beta t ; \alpha, \beta, \delta$ are determined in (44); $A_{1}, C_{1}$, and $C_{2}$, and $\nu$ are arbitrary constants.

Consider

$$
\begin{aligned}
& w_{17}(x, t) \\
& =\frac{A_{1} \sqrt{-\delta}\left(C_{1} \sinh (\sqrt{-\delta} \xi)+C_{2} \cosh (\sqrt{-\delta} \xi)\right)}{C_{1} \cosh (\sqrt{-\delta} \xi)+C_{2} \sinh (\sqrt{-\delta} \xi)},
\end{aligned}
$$

where $\xi=\alpha x+\beta t ; \alpha, \beta$ are determined in (45); $A_{1}, C_{1}, C_{2}$, and $\delta$ are arbitrary constants.

Consider

$$
\begin{aligned}
w_{18}(x, t) & \\
= & \frac{A_{1} \sqrt{-\delta}\left(C_{1} \sinh (\sqrt{-\delta} \xi)+C_{2} \cosh (\sqrt{-\delta} \xi)\right)}{C_{1} \cosh (\sqrt{-\delta} \xi)+C_{2} \sinh (\sqrt{-\delta} \xi)} \\
& +\frac{B_{1}}{C_{1} \cosh (\sqrt{-\delta} \xi)+C_{2} \sinh (\sqrt{-\delta} \xi)},
\end{aligned}
$$

where $\xi=\alpha x+\beta t ; \alpha, \beta, \delta$ are determined in (46); $A_{1}, B_{1}$, and $C_{1}$, and $C_{2}$ are arbitrary constants.

If $\delta=0$, one has

$$
\begin{gathered}
A_{0}=0, \quad A_{1}=A_{1}, \quad B_{1}=B_{1}, \quad \alpha=-\frac{1}{3} c \epsilon \lambda_{3} A_{1}, \\
\beta=-\frac{1}{6} c^{2} \epsilon \lambda_{3} A_{1}, \quad \nu=\frac{A_{1}^{2} C_{1}^{2}-B_{1}^{2}}{2 A_{1}^{2} C_{2}}, \\
A_{0}=0, \quad A_{1}=A_{1}, \quad B_{1}=0, \quad \alpha=-\frac{1}{6} c \epsilon \lambda_{3} A_{1}, \\
\beta=-\frac{1}{12} c^{2} \in \lambda_{3} A_{1}, \quad v=0 .
\end{gathered}
$$

Substituting (51) and (52) into (34), making use of solutions (15) of (14), we can obtain, respectively, exact traveling wave solutions expressed by rational functions of (2).

Consider

$$
w_{19}(x, t)=\frac{2 A_{1}\left(\nu \xi+C_{1}\right)}{\nu \xi^{2}+2\left(C_{1} \xi+C_{2}\right)}+\frac{2 B_{1}}{\nu \xi^{2}+2\left(C_{1} \xi+C_{2}\right)},
$$

where $\xi=\alpha x+\beta t ; \alpha, \beta$, and $\nu$ are determined in (51); $B_{1}, C_{1}$, and $C_{2}$ are arbitrary constants.

Consider

$$
w_{20}(x, t)=\frac{A_{1} C_{1}}{C_{1} \xi+C_{2}},
$$

where $\xi=\alpha x+\beta t ; \alpha$ and $\beta$ are determined in (52); $A_{1}, C_{1}$, and $C_{2}$ are arbitrary constants.

\section{Conclusions}

In [28] Rees derived a new nonlinear KdV-like evolution equation (2) for surface solitary waves propagating on a liquid-air interface where the wave motion is induced by harmonic forcing. The study on the exact solutions for (2) is meaningful and important. However, exact traveling wave solutions of (2) are not reported in related references. In this work, we apply the simplest equation method, the modified simplest equation method, and the extended simplest equation method for obtaining exact traveling solutions of (2). And some new exact solutions expressed by hyperbolic 
function, trigonometric function, and rational functions are obtained. The correctness of all the solutions is verified by substituting them into original equation (2). It is easy to see that the simplest equation methods are direct, concise, effective, and reliable, which can be used for many other NLEEs in mathematical physics.

\section{Conflict of Interests}

The authors declare that there is no conflict of interests regarding the publication of this paper.

\section{Acknowledgments}

This research is supported by the Natural Science Foundation of China (nos. 11161020 and 11361023) as well as the Young and Middle-Aged Academic Backbone of Honghe University (no. 2014GG0105).

\section{References}

[1] M. J. Ablowitz and P. A. Clarkson, Solitons, Nonlinear Evolution Equations and Inverse Scattering, Cambridge University Press, New York, NY, USA, 1991.

[2] V. B. Matveev and M. A. Salle, Darboux Transformations and Solitons, Springer, Berlin, Gemany, 1991.

[3] R. Hirota, "Exact solution of the korteweg-de vries equation for multiple Collisions of solitons," Physical Review Letters, vol. 27, no. 18, pp. 1192-1194, 1971.

[4] M. Wang, Y. Zhou, and Z. Li, "Application of a homogeneous balance method to exact solutions of nonlinear equations in mathematical physics," Physics Letters, Section A: General, Atomic and Solid State Physics, vol. 216, no. 1-5, pp. 67-75, 1996.

[5] S. Liu, Z. Fu, and Q. Zhao, "Jacobi elliptic function expansion method and periodic wave solutions of nonlinear wave equations," Physics Letters A, vol. 289, no. 1-2, pp. 69-74, 2001.

[6] E. J. Parkes and B. R. Duffy, "An automated tanh-function method for finding solitary wave solutions to non-linear evolution equations," Computer Physics Communications, vol. 98, no. 3, pp. 288-300, 1996.

[7] J. He and X. Wu, "Exp-function method for nonlinear wave equations," Chaos, Solitons and Fractals, vol. 30, no. 3, pp. 700708, 2006.

[8] M. Wang, X. Li, and J. Zhang, "The $G^{\prime} / G$-expansion method and travelling wave solutions of nonlinear evolution equations in mathematical physics," Physics Letters. A, vol. 372, no. 4, pp. 417-423, 2008.

[9] S. Guo and Y. Zhou, "The extended $G^{\prime} / G$-expansion method and its applications to the Whitham-Broer-Kaup-like equations and coupled Hirota-Satsuma KDV equations," Applied Mathematics and Computation, vol. 215, no. 9, pp. 3214-3221, 2010.

[10] M. V. Demina and N. A. Kudryashov, "From Laurent series to exact meromorphic solutions: the Kawahara equation," Physics Letters A, vol. 374, no. 39, pp. 4023-4029, 2010.

[11] M. V. Demina and N. A. Kudryashov, "Explicit expressions for meromorphic solutions of autonomous nonlinear ordinary differential equations," Communications in Nonlinear Science and Numerical Simulation, vol. 16, no. 3, pp. 1127-1134, 2011.
[12] N. Jamshidi and D. D. Ganji, "Application of energy balance method and variational iteration method to an oscillation of a mass attached to a stretched elastic wire," Current Applied Physics, vol. 10, no. 2, pp. 484-486, 2010.

[13] M. Rafei, H. Daniali, and D. D. Ganji, "Variational interation method for solving the epidemic model and the prey and predator problem," Applied Mathematics and Computation, vol. 186, no. 2, pp. 1701-1709, 2007.

[14] F. Fouladi, E. Hosseinzadeh, A. Barari, and G. Domairry, "Highly nonlinear temperature-dependent fin analysis by variational iteration method," Heat Transfer Research, vol. 41, no. 2, pp. 155-165, 2010.

[15] M. Esmaeilpour and D. D. Ganji, "Application of He's homotopy perturbation method to boundary layer flow and convection heat transfer over a flat plate," Physics Letters A, vol. 372, no. 1, pp. 33-38, 2007.

[16] D. D. Ganji, M. J. Hosseini, and J. Shayegh, "Some nonlinear heat transfer equations solved by three approximate methods," International Communications in Heat and Mass Transfer, vol. 34, no. 8, pp. 1003-1016, 2007.

[17] Z. Z. Ganji, D. D. Ganji, and M. Esmaeilpour, "Study on nonlinear Jeffery-Hamel flow by He's semi-analytical methods and comparison with numerical results," Computers \& Mathematics with Applications, vol. 58, no. 11-12, pp. 2107-2116, 2009.

[18] S. H. H. Nia, A. N. Ranjbar, D. D. Ganji, H. Soltani, and J. Ghasemi, "Maintaining the stability of nonlinear differential equations by the enhancement of HPM," Physics Letters A: General, Atomic and Solid State Physics, vol. 372, no. 16, pp. 2855-2861, 2008.

[19] M. Omidvar, A. Barari, M. Momeni, and D. Ganji, "New class of solutions for water infiltration problems in unsaturated soils," Geomechanics and Geoengineering, vol. 5, no. 2, pp. 127-135, 2010.

[20] E. Fan, "Uniformly constructing a series of explicit exact solutions to nonlinear equations in mathematical physics," Chaos, Solitons and Fractals, vol. 16, no. 5, pp. 819-839, 2003.

[21] N. A. Kudryashov, "Exact solitary waves of the Fisher equation," Physics Letters A, vol. 342, no. 1-2, pp. 99-106, 2005.

[22] N. A. Kudryashov, "Simplest equation method to look for exact solutions of nonlinear differential equations," Chaos, Solitons and Fractals, vol. 24, no. 5, pp. 1217-1231, 2005.

[23] A. J. Mohamad Jawad, M. D. Petkovic, and A. Biswas, "Modified simple equation method for nonlinear evolution equations," Applied Mathematics and Computation, vol. 217, no. 2, pp. 869$877,2010$.

[24] N. A. Kudryashov and N. B. Loguinova, "Extended simplest equation method for nonlinear differential equations," Applied Mathematics and Computation, vol. 205, no. 1, pp. 396-402, 2008.

[25] E. M. E. Zayed and S. A. H. Ibrahim, "Exact solutions of nonlinear evolution equations in mathematical physics using the modified simple equation method," Chinese Physics Letters, vol. 29, no. 6, Article ID 060201, 2012.

[26] S. Bilige and T. Chaolu, "An extended simplest equation method and its application to several forms of the fifth-order $\mathrm{KdV}$ equation," Applied Mathematics and Computation, vol. 216, no. 11, pp. 3146-3153, 2010.

[27] S. Bilige, T. Chaolu, and X. Wang, "Application of the extended simplest equation method to the coupled SchrödingerBoussinesq equation," Applied Mathematics and Computation, vol. 224, pp. 517-523, 2013. 
[28] J. M. Rees and W. B. Zimmerman, "An intermediate wavelength, weakly nonlinear theory for the evolution of capillary gravity waves," Wave Motion, vol. 48, no. 8, pp. 707-716, 2011.

[29] A. Sohail, J. M. Rees, and W. B. Zimmerman, "Analysis of capillary-gravity waves using the discrete periodic inverse scattering transform," Colloids and Surfaces A: Physicochemical and Engineering Aspects, vol. 391, no. 1-3, pp. 42-50, 2011.

[30] A. Sohail, K. Maqbool, and T. Hayat, "Painlevé property and approximate solutions using Adomian decomposition for a nonlinear KdV-like wave equation," Applied Mathematics and Computation, vol. 229, pp. 359-366, 2014. 


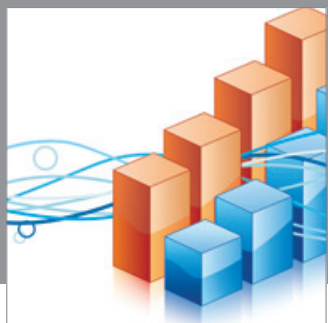

Advances in

Operations Research

mansans

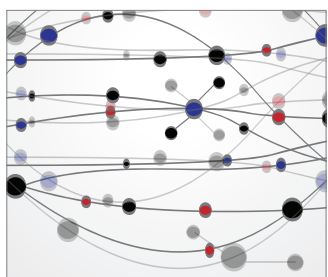

The Scientific World Journal
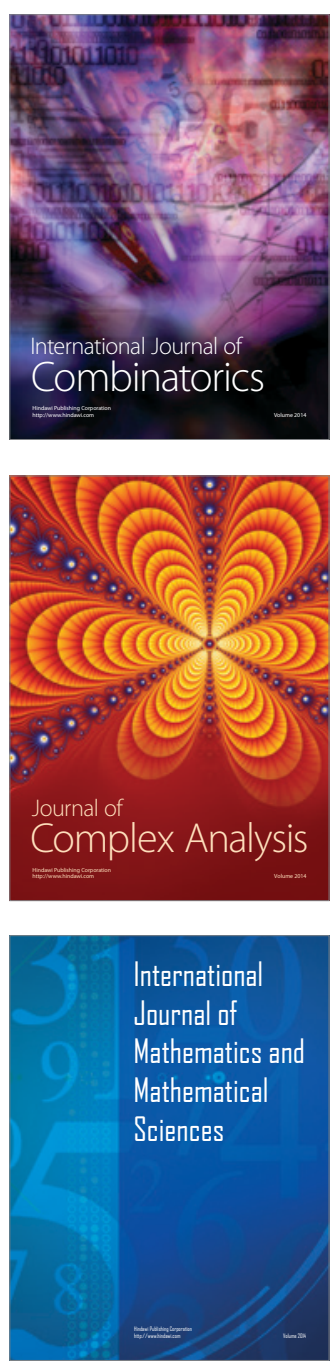
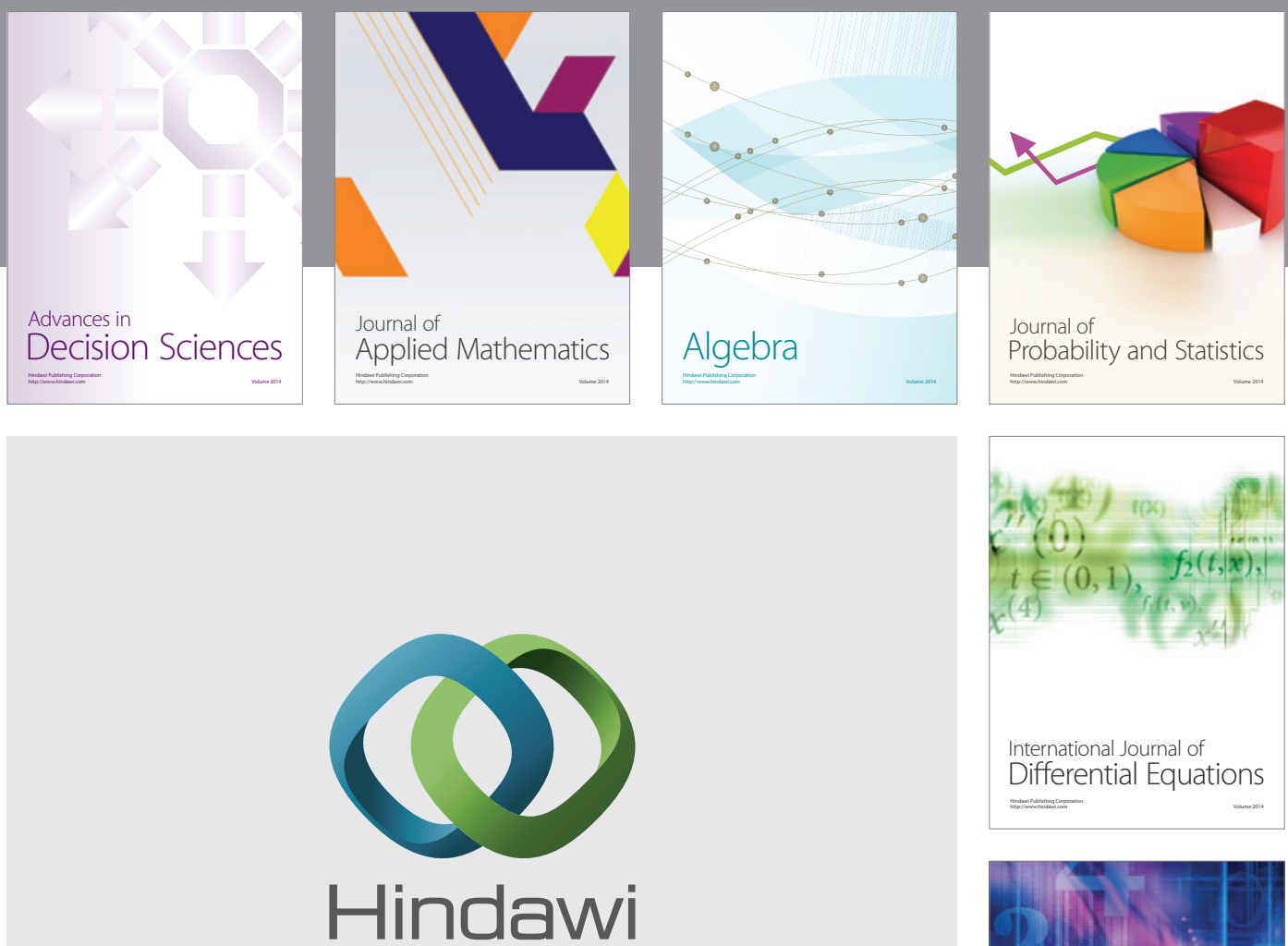

Submit your manuscripts at http://www.hindawi.com
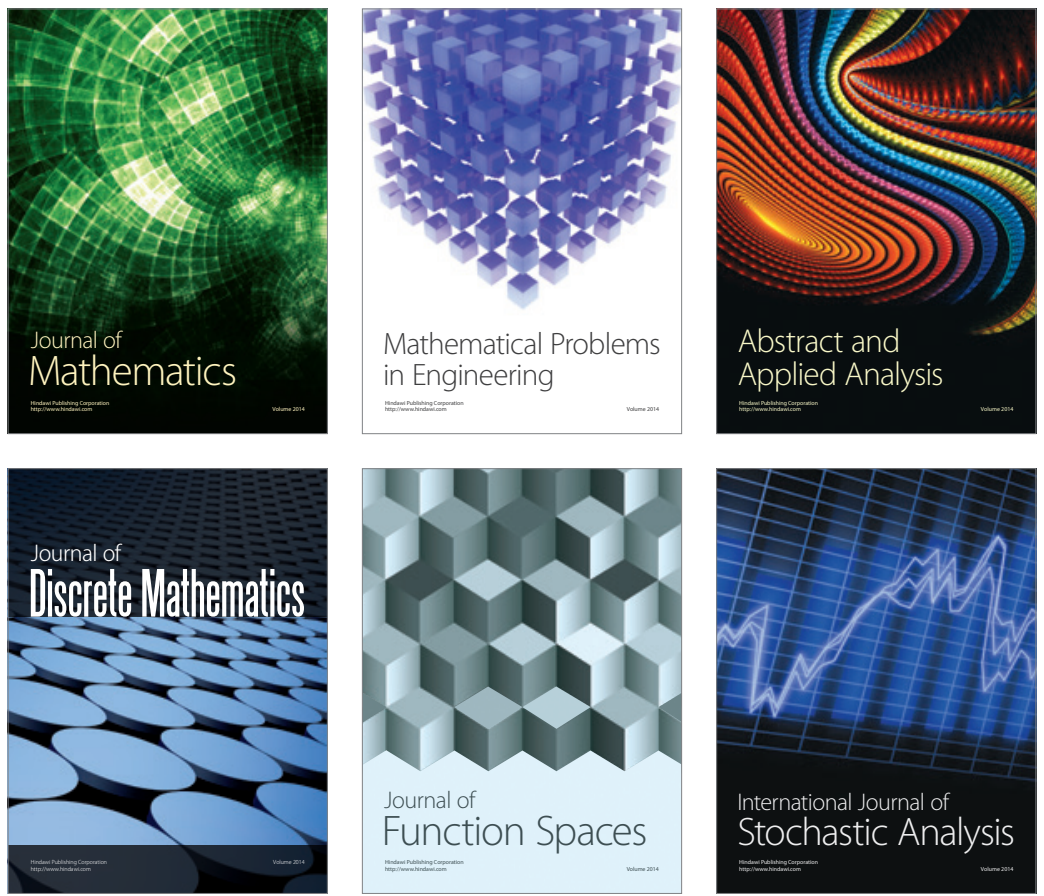

Journal of

Function Spaces

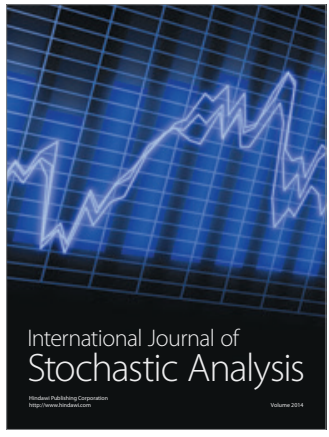

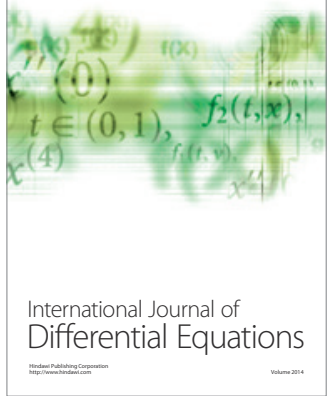
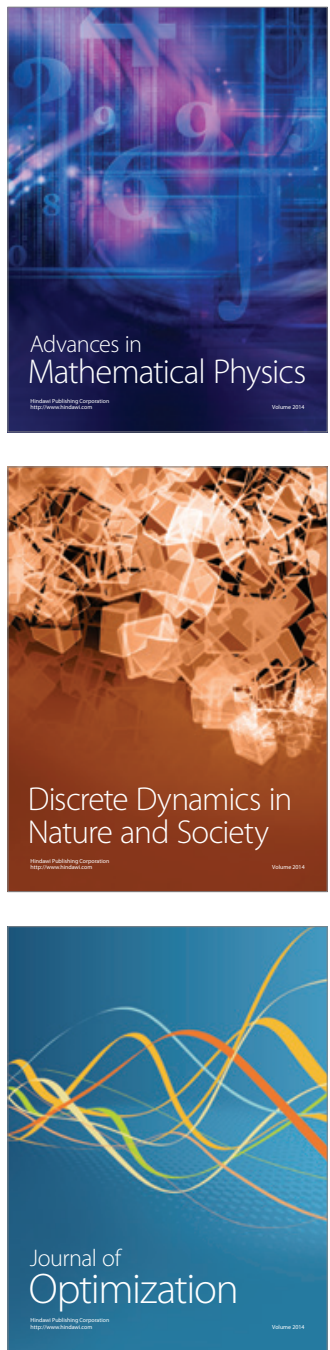